Full Paper

\title{
Önologische und sensorische Charakterisierung der Rebsorten Fraueler, Versoalen, Weissterlaner, Blatterle und Furner
}

\author{
Enological and sensorial characterisation of the varietys Fraueler, Versoalen, Weissterlaner, Blatterle \\ and Furner
}

Caratterizzazione enologica e sensoriale delle varietà Fraueler, Versoalen, Weissterlaner, Blatterle und Furner

Christoph Patauner ${ }^{1}$, Ulrich Pedri ${ }^{1}$, Josef Terleth ${ }^{1}$, Eva Überegger ${ }^{1}$

1 Versuchszentrum Laimburg

\section{ABSTRACT}

South Tyrol has always been a land of many different grape varieties. Many varieties have been cultivated over the years but also abandoned again. However, there is still a niche market in South Tyrol for old, historic local varieties, of which there is a remnant stock that is vinified as a single variety or in a blend. However, some of these varieties do not appear in any catalog of varieties. This project will be used to prepare a dossier for the varieties Fraueler, Versoalen, Weissterlaner, Blatterle and Furner in order to register them in the National Variety Catalog.

\section{KEYWORDS}

Fraueler, Versoalen, Weissterlaner, Blatterle, Furner

\section{CITE ARTICLE AS}

Patauner Christoph, Pedri Ulrich, Terleth Josef et.al. (2019).

Enological and sensorial characterisation of the varietys Fraueler, Versoalen, Weissterlaner, Blatterle and Furner. Laimburg Journal 1/2019

DOI: $10.23796 / \mathrm{LJ} / 2019.005$

\section{CORRESPONDING AUTHOR}

Christoph Patauner

Laimburg 6, Pfatten, I-39040 Auer (BZ), Italien

christoph.patauner@laimburg.it +390471969626 


\section{EINFÜHRUNG}

Südtirol ist seit jeher ein Land der vielen verschiedenen Rebsorten. Viele Sorten sind im Laufe der Jahre aus den verschiedensten Gründen angebaut aber auch wieder entfernt worden. Nach wie vor gibt es aber auch in Südtirol einen Nischenmarkt für alte historische Lokalsorten, von denen ein Restbestand besteht und die entweder reinsortig oder im Verschnitt ausgebaut werden.

Im Rahmen dieses Projektes wurden Trauben dieser Sorten zu Wein verarbeitet. Die Weine wurden sowohl analytisch als auch sensorisch charakterisiert.

\section{METHODEN}

Zwischen 2014 und 2016 wurden Trauben aus verschiedenen Standorten der Sorten Fraueler, Versoalen, Weissterlaner, Blatterle und Furner zu Wein verarbeitet. Die Trauben der Sorte Blatterle stammten aus einer Anlage in Bozen - Bozner Boden. Die FurnerTrauben kamen vom Putzerhof in Lajen während hingegen die Fraueler aus einer Anlage in Schlanders - Vetzan stammen. Versoalen und Weissterlaner stammen direkt vom Standort Stadlhof - Laimburg. Da die oben genannten Rebsorten zum größten Teil sehr schwache Mostgewichte erbrachten, wurden Moste bzw. Maischen wie in der nachstehend angeführten Tabelle 1 aufgezeigt wird mit rektifiziertem Traubenmostkonzentrat angereichert, um den späteren Alkoholgehalt auf ein für Qualitätsweine zutreffendes Mindestmaß anzuheben. In der Tabelle 2 sind die einzelnen Lesezeitpunkte ersichtlich.

\section{WEIßWEINBEREITUNG}

\section{VERSOALEN, BLATTERLE, WEISSTERLANER,} FRAUELER

Die Trauben wurden zum entsprechenden Lesezeitpunkt, welcher aus Tabelle 2 hervorgeht, von Hand geerntet und in Kisten mit 18 kg Fassungsvermögen zur Weiterverarbeitung in den Kellereibetrieb des Versuchszentrums Laimburg gebracht. Nach klassicher Weißweinbereitung vinifiziert, wurde somit auf eine Maischestandzeit verzichtet. Als Abbeermaschine kam das Model Lugana $1 R$ des Herstellers CMA zum Einsatz (Stundenleistung 4-6 t/h). Die Trauben wurden nach Entnahme der Stachelwalze leicht angequetscht jedoch nicht vom Stielgerüst abgebeert. Das angequetschte Traubenmaterial wurde daraufhin händisch in die vollau- tomatisch betriebenen Pressen des Typs Europress Modell T1 mit 100 I Fassungsvermögen gegeben und mit einem Automatikprogramm gepresst. Es kamen dabei 2 verschiedene Druckstufen zum Einsatz. Begonnen wurde mit einem Druck von 1 bar für $10 \mathrm{Mi}$ nuten, worauf zwei Wiederholungen von je 10 Minuten mit 2 bar Druck folgten. Der Rücksetzdruck belief sich dabei immer auf 0.05 bar. Zwischen jeder Druckstufe wurde der aufgebaute Druck abgebaut, ausreichend gekrümelt und mit der nächsten Druckstufe fortgefahren. Der ablaufende Saft wurde zur Gänze in der Mostauffangwanne gesammelt, wobei $40 \%$ der zu erwartenden Zielmenge unverzüglich mit $30 \mathrm{mg} / \mathrm{l}$ Kaliummetabisulfit (E 224) behandelt wurden. Erst nachdem der automatische Presszyklus beendet war, wurde der Saft in einer Korbflasche mit 34 I Fassungsvermögen gesammelt und die Gesamtmenge nun definitiv mit $30 \mathrm{mg} / \mathrm{l}$ Kaliummetabisulfit ( $\mathrm{E}$ 224) behandelt bzw. nachgeschwefelt.

Wie in der klassischen Weißweinbereitung üblich, wurde der Most entschleimt, um inn von Trub- und Schmutzstoffen zu trennen. Damit ermöglicht man eine saubere Gärung und hilft, Fehlaromen zu vermeiden. Dies passierte in diesem Versuch mit der statischen Methode. Dabei wurden die Moste einfach für 16 Stunden bei $5{ }^{\circ} \mathrm{C}$ gelagert und entschleimten somit statisch. Im Anschluss wurde der klare Überstand abgezogen und der Entschleimungstrub entfernt. Vorsichtig auf $20{ }^{\circ} \mathrm{C}$ erwärmt, wurde je Ausbau eine Mostprobe entnommen, um diese dann im Weinlabor des Versuchszentrums Laimburg auf die wichtigsten Mostinhaltsstoffe untersuchen zu lassen. Um eine sichere und zügige Gärung zu gewährleisten, wurden Trockenreinzuchthefe der Art Saccharomyces Cerevisiae Var. Cerevisiae verschiedener Stämme eingesetzt. Die Hefepräparate wurden nach Angabe des Herstellers rehydriert und dann dem Most zugesetzt. Die anschließende alkoholische Gärung erfolgte bei konstanten $21^{\circ} \mathrm{C}$ über regulierte Raumtemperatur. Die Gärdauer war je nach Sorte, Jahrgang und Standort individuell. Der Abstich erfolgte immer bei Gärungsstillstand bzw. bei Restzuckergehalt $<4.0 \mathrm{~g} / \mathrm{l}$. Der erste $\mathrm{Ab}$ stich erfolgte immer unbelüftet.

Um instabile Weinsäure auszufällen, folgte nach dem ersten Abzug eine kurzzeitige Lagerung in der Kühlzelle bei $5{ }^{\circ} \mathrm{C}$ für ca. 10 Tage. Anschließend wurden die Jungweine mit 30 mg/l Kaliummetabisulfit (E 224) geschwefelt. Nach einem weiteren Abzug la- gerten die Weine bei einer Temperatur zwischen 14 und $18{ }^{\circ} \mathrm{C}$. Dabei wurde ein Gehalt an freier schwefeliger Säure von $25 \mathrm{mg} / \mathrm{l}$ eingestellt und überwacht. Die Füllung erfolgte in 0.5 I Glasflaschen mit 30/60 BVS Mündung nach unmittelbar vorhergehender VorBlank- und Sterilfiltration $(0.45 \mu \mathrm{m})$. Als Verschluss wurde Stelvin 30/60 BVS, Dichtungsscheibe Stanniol, gewählt.

Vom „Labor für Wein- und Getränkeanalytik des Versuchszentrums Laimburg" wurden auf Wein Analysen im Hinblick auf folgende Inhaltsstoffe durchgeführt:
- Alkohol im Wein \%vol. (FOSS ${ }^{\circledR}$, Wine- $\left.\mathrm{Scan}^{\mathrm{TM}} \quad \mathrm{SO} 2\right), \quad$ Methodenbuch 5.04pp45rev.0

- Gesamtsäure im Wein g/l (FOSS ${ }^{\circledR}$, Wine$\left.\mathrm{Scan}^{\mathrm{TM}} \quad \mathrm{SO} 2\right), \quad$ Methodenbuch $5.04 \mathrm{pp} 45 \mathrm{rev} .0$

- Gesamtextrakt im Wein g/l (FOSS ${ }^{\circledR}$, Wine$\mathrm{Scan}^{\mathrm{TM}}$ SO2), Methodenbuch 5.04pp45rev.0

- Weinsäure im Wein g/l, Methodenbuch $5.04 \mathrm{pp} 46 \mathrm{rev} .0$

- Apfelsäure im Wein g/l, Methodenbuch 5.04pp51 rev.0

- Gesamtpolyphenole im Wein mg/l, Methodenbuch 5.04pp17 rev.1

Alle Weine wurden im Folgejahr der Vinifizierung einer sensorischen Prüfung unterzogen. Die sensorisch zu diskriminierenden Kriterien sind in Tabelle 3 angeführt. Als Verkostungsprofil diente dabei eine Vorlage nach Weiß (1972) [1], welche in Abbildung 1 ersichtlich ist. Um die Urteilssicherheit der einzelnen Panelmitglieder (Techniker, Berater und Fachleute aus Weinbau und Kellerwirtschaft) zu überprüfen wurde das Verfahren nach Kobler (1996) [2] angewandt, wobei für jeden einzelnen Parameter die Kontrollalgorithmen durchgerechnet wurden. Für die Auswertung der erhobenen Daten wurden die Programme Microsoft ${ }^{\circledR}$ Exce 2016 MSO (16.0.8201.2209) 32-Bit und IBM $^{\circledR}$ SPSS $^{\circledR}$ Statistics Version 20 Release 20.0.0 für varianzanalytische Mittelwertvergleiche (Oneway ANOVA) oder multivarianter Varianzanalyse (Manova) eingesetzt. Als Post-Hoc-Test wurde Tukey-B mit Signifikanzniveau 0.05 über SPSS gerechnet. 


\section{ROTWEINBEREITUNG}

\section{FURNER}

Die Trauben wurden zum entsprechenden Lesezeitpunkt, welcher aus Tabelle 2 hervorgeht, von Hand geerntet und in Kisten mit 18 kg Fassungsvermögen zur Weiterverarbeitung in den Kellereibetrieb des Versuchszentrums Laimburg gebracht. Um das Stielgerüst von den Beeren zu trennen wurden die Trauben mit einer Abbeermaschine des Typs CMA Lugana $1 R$ (Stundenleistung 4-6 $\mathrm{t} / \mathrm{h}$ ) abgebeert. Daraufhin wurden Maischen in Weithalskorbflaschen mit einem Fassungsvermögen von 34 I gegeben und mit 30 mg/l Kaliummetabisulfit (E 224) geschwefelt. Anschließend wurde je Ausbau eine Mostprobe entnommen, um diese im Weinlabor des Versuchszentrums Laimburg auf die wichtigsten Mostinhaltsstoffe zu untersuchen. Um eine sichere und zügige Gärung zu gewährleisten, wurden Trockenreinzuchthefen der Art Saccharomyces Cerevisiae Var. Cerevisiae verschiedener Stämme eingesetzt. Die Hefepräparate wurden nach Angabe des Herstellers rehydriert und dann der Maische zugesetzt. Die alkoholische Gärung erfolgte bei konstanten $23.5^{\circ} \mathrm{C}$ über regulierte Raumtemperatur, wobei die Gärdauer je nach Jahrgang unterschiedlich war. Der Abzug von den Trestern erfolgte bei Gärungsstillstand bzw. bei Restzuckergehalt < $4.0 \mathrm{~g} / \mathrm{l}$. Dieser erste Abstich erfolgte mit starker Belüftung über ein Kupfersieb. Die Trester wurden mit vollautomatisch betriebenen Pressen des Typs Europress Modell T1 mit 100 | Fassungsvermögen bei einem Druck von 0.5 bar (Rücksetzdruck 0.05 bar) für 10 Minuten schonend ausgepresst. Der dadurch gewonnene Druckwein wurde der Gesamtmenge zugeführt. Zwei Tage später wurden die Jungweine vom Hefegeläger abgezogen und in Korbflaschen spundvoll bei $23.5^{\circ} \mathrm{C}$ gelagert. Sobald der spontan gestartete biologische Säureabbau abgeschlossen war, wurden die Weine erneut abgezogen und mit 20 mg/l Kaliummetabisulfit (E 224) geschwefelt. Anschließend folgte eine Lagerung zwischen 14 und $18{ }^{\circ} \mathrm{C}$. Während der Weinlagerung wurde ein Gehalt an freier schwefeliger Säure von $25 \mathrm{mg} / \mathrm{l}$ eingestellt und überwacht. Die Füllung erfolgte in 0.5 I Glasflaschen mit 30/60 BVS Mündung nach unmittelbar vorhergehender Vor-, Blankund Sterilfiltration $(0.45 \mu \mathrm{m})$. Als Verschluss wurde Stelvin 30/60 BVS, Dichtungsscheibe Stanniol, gewählt.

Vom „Labor für Wein- und Getränkeanalytik des Versuchszentrums Laimburg" wurden die Weine auf folgende Inhaltsstoffe analysiert:

- Alkohol im Wein \%vol. (FOSS ${ }^{\circledR}$, Wine$\mathrm{Scan}^{\mathrm{TM}}$ SO2), Methodenbuch 5.04pp45rev.0

- Gesamtsäure im Wein g/l (FOSS ${ }^{\circledR}$, Wine$\left.\mathrm{Scan}^{\mathrm{TM}} \quad \mathrm{SO} 2\right), \quad$ Methodenbuch 5.04pp45rev.0

- Gesamtextrakt im Wein g/l (FOSS ${ }^{\circledR}$, Wine$\mathrm{Scan}^{\mathrm{TM}}$ SO2), Methodenbuch 5.04pp45rev.0

- Weinsäure im Wein $\mathrm{g} / \mathrm{l}$, Methodenbuch $5.04 \mathrm{pp} 46 \mathrm{rev} .0$

- Milchsäure im Wein g/l, Methodenbuch $5.04 p p 46$ rev.0

- Gesamtpolyphenole mg/l, Methodenbuch $5.04 \mathrm{pp} 17$ rev.1

- Anthocyane mg/l, Methodenbuch $5.04 \mathrm{pp} 02$ rev.0

Die Weine der Rebsorte Furner wurden rund 16 Monate nach der Vinifizierung einer sensorischen Prüfung unterzogen. Die sensorisch zu diskriminierenden Kriterien sind in Tabelle 4 angeführt. Als Verkostungsprofil diente dabei eine Vorlage nach Weiß (1972) [1], welche in Abbildung 1 ersichtlich ist. Um die Urteilssicherheit der einzelnen Panelmitglieder (Techniker, Berater und Fachleute aus Weinbau und Kellerwirtschaft) zu überprüfen wurde das Verfahren nach Kobler (1996) [2] angewandt, wobei für jeden einzelnen Parameter die Kontrollalgorithmen durchgerechnet wurden. Für die Auswertung der erhobenen Daten wurden die Programme Microsoft ${ }^{\circledR}$ Excel 2016 MSO (16.0.8201.2209) 32-Bit und IBM $^{\circledast}$ SPSS $^{\circledast}$ Statistics Version 20 Release 20.0.0 für varianzanalytische Mittelwertvergleiche (Oneway ANOVA) oder multivarianter Varianzanalyse (Manova) eingesetzt. Als PostHoc-Test wurde Tukey-B mit Signifikanzniveau 0.05 über SPSS gerechnet.

\section{ERGEBNISSE}

\section{BLATTERLE}

Die auch in frühen Lagen eher spät ausreifende Sorte Blatterle erbringt in den meisten Fällen die Mindestanforderung für Qualitätswein (Tab. 5).

Sensorisch gesehen ist Blatterle eine eher einfache Sorte mit mäßig fülligen Weinen. Das Aroma wird vordergründig von Kernobst dominiert, dahinter findet sich etwas an exotischer Frucht und Steinobst sowie auch leicht grünliche Noten (Abb. 2). Wie Abbildung 3 zeigt, erinnert Blatterle hauptsächlich an einen Burgundertyp.

\section{FRAUELER}

Die Sorte Fraueler entspricht analytisch gesehen den gängigen Parametern für Qualitätswein. Auffallend sind die hohe Gesamtsäure und der niedrige pH-Wert (Tab. 6).

Sensorisch gesehen ist der Wein sehr einfach, mit leichtem Körper und wenig Fülle. Es dominiert die Zitrusfrucht, dahinter etwas grüne Aromen und Blüten (Abb. 4). Vom Typ her ist Fraueler nicht eindeutig zuordenbar (Abb. 5).

\section{WEISSTERLANER}

Die Sorte Weissterlaner erfüllt alle gängigen Mindeststandards für Qualitätswein. Zu erwähnen ist der etwas geringe Alkoholgehalt (Tab. 7).

Weissterlaner erbringt einfache, neutrale, aber recht füllige Weine hervor. Die Aromatik ist deutlich von Kernobst geprägt, dahinter etwas exotische Früchte, Blüten und $\mathrm{Heu}$ (Abb. 6). Weissterlaner ist am meisten mit einem klassischen Burgundertyp zu vergleichen (Abb. 7).

\section{VERSOALEN}

Versoalen ist eine eher spät reifende Weißweinsorte. Sie erfüllt alle gängigen Standards für Qualitätswein (Tab. 8).

Versoalen bringt mittelgewichtige und eher neutrale Weine hervor, die sich am Typ eines Burgunders orientieren (Abb. 9). Es dominieren Kernobst und Zitrusfrucht, dahinter exotische Früchte und etwas Steinobst (Abb. 8).

\section{FURNER}

Die Rotweinsorte Furner erbringt alle Mindestanforderungen für Qualitätswein. Auffallend ist der stark variierende Gehalt an Gesamtpolyphenolen (Tab. 9).

Weine aus Furner-Trauben sind recht fruchtbetonte Weine. Es dominieren Kirsche, Beerenobst und Waldfrucht (Abb. 10). Die Farbe bewegt sich im rubinroten Bereich mit stark violetten Reflexen. Anzuführen ist der wenig vorhandene Gerbstoff. Furner ähnelt einem Vernatsch-Wein (Abb. 11).

Abbildung 12 gibt einen Überblick über die Gerbstoffquantität von Furner im Vergleich mit klassischen Südtiroler Rotweinsorten. 


\section{DISKUSSION}

Klassisch ausgebaut sind die Rebsorten Fraueler, Versoalen, Weissterlaner, Blatterle und Furner im Keller grundsätzlich einfach zu handhaben. Bei den fertig abgefüllten Weinen zeigt sich allerdings immer wieder das mäßige Qualitätspotential dieser Sorten, weshalb vielleicht in Zukunft andere Vinifizierungsmethoden in Frage kommen könnten wie z.B. die Versektung. Als Nischenprodukte werden diese sogenannten historischen Rebsorten Südtirols sicherlich ihren Platz behalten und stellen somit eine wichtige Facette der Südtiroler Weinlandschaft dar.

\section{DANKSAGUNG}

Wir möchten uns bei dieser Gelegenheit bei Familie Schuster (Befehlhof Vetzan), Heinrich Mayr (Nusserhof Bozen) und Hubert Runggatscher (Putzerhof Lajen Ried) für die Zurverfügungstellung von Traubenmaterial recht herzlich bedanken. Weiters danken wir allen Mitarbeiterinnen und Mitarbeitern des Versuchszentrums Laimburg, die an der Umsetzung und Ausführung dieses Projektes beteiligt waren.

\section{ZUSAMMENFASSUNG}

Südtirol ist seit jeher ein Land der vielen verschiedenen Rebsorten. Viele Sorten sind im Laufe der Jahre aus den verschiedensten Gründen angebaut aber auch wieder entfernt worden. Nach wie vor gibt es aber auch in Südtirol einen Nischenmarkt für alte historische Lokalsorten, von denen ein Restbestand besteht und die entweder reinsortig oder im Verschnitt ausgebaut werden. Allerdings scheinen einige dieser Sorten in keinem Sortenkatalog auf. Dieses Projekt dient zur Ausarbeitung eines Dossiers für die Sorten Fraueler, Versoalen, Weissterlaner, Blatterle und Furner, um diese in den Nationalen Rebsortenkatalog eintragen zu lassen..

\section{RIASSUNTO}

Il Sudtirolo è da sempre un paese dagli innumerevoli vitigni. Molti di loro nel corso degli anni sono stati coltivati per vari motivi e alcuni sono anche scomparsi. Tuttavia esiste un mercato di nicchia per vecchie varietà locali, dei quali esiste una quantità marginale $e$ che vengono vinificati o in purezza oppure in unvaggio. Alcuni di loro non risultano in nessun catalogo varietale nazionale. Questo progetto serve per la stesura di un dossier per le Varietà Fraueler, Versoaln, Weissterlaner, Blatterle e Furner, per poter registrarle nel catalogo nazionale delle varietà. 


\section{LITERATUR}

[1] Weiss J., Willisch E., Knorr D., Schaller A.

(1972). Ergebnisse von Untersuchungen bezüglich der differenzierten Wirkung einer sensorischen bewertenden Prüfmethode gegenüber einer sensorischen Rangordnungs-

[2] Kobler A. (1996). La valutazione sensoriale dei vini ed il controllo degli assaggiatori mediante l'uso di schede di degustazione non strutturate. Rivista di Viticoltura e di EnoloPrüfmethode am Beispiel von Apfelsaft und gia 49(4), 3-18.

Birnennektar. Confructa 17(4/5), 237-250. 


\section{ANHANG 1: ABBILDUNGEN}

\author{
Koster
}

D atu m

…….................
Gruppe

Weinnum $m$ er
Harmonie (Säure, Alkohol, Tannin)
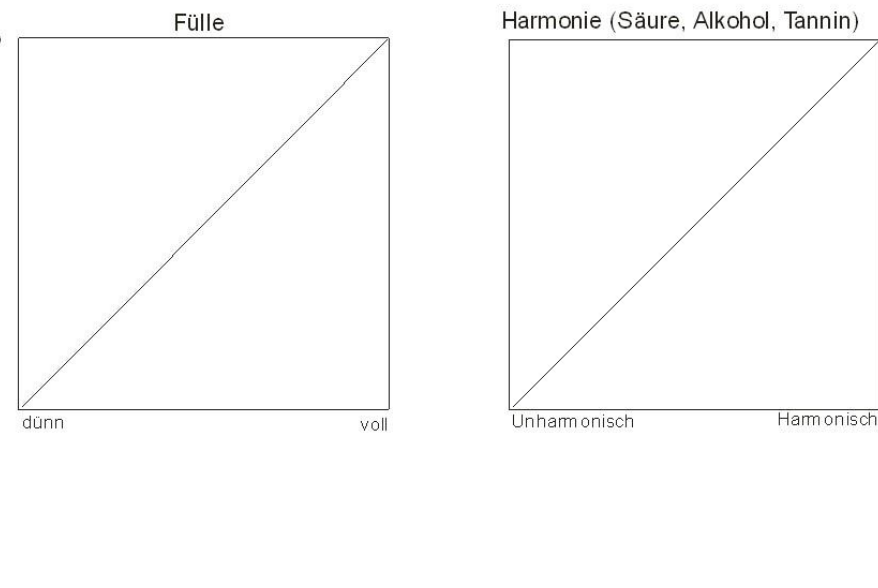

Grundfarbe

Reflexe

Farbe

Kernobst (Apfel, Bime)

Hellgelb

Gelb

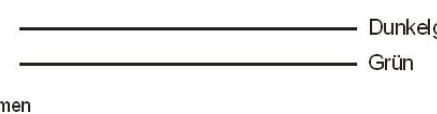

Exotische Frucht (Ananas, Banane) nicht vorh.

Steinobst (Pfirsich, Aprikose)

Blüten (Rosen, Veilchen, Nelken)

Gewürze (Pfeffer, Muskat)

nicht vorh.

nicht vorh.

nicht vorh.

nicht vorh.

Zitrusfrucht (Zitrone, Pompelmo)

Grüne Aromen (Brennessel, Paprika) nicht vorh.

Honig

nicht vorh.

nicht vorh.

nicht vorh.

Litschi

nicht vorh.

nicht vorh.

vorhanden

Kräuter
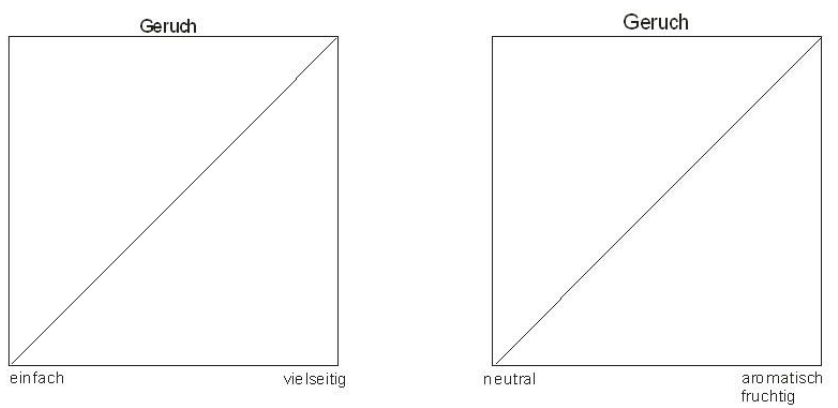

Burgundertyp

Rieslingtyp

Gewürztraminertyp

Sauvignontyp

Muskattyp

andere:

Abb. 1: Verkostungsbogen // tasting notes. 


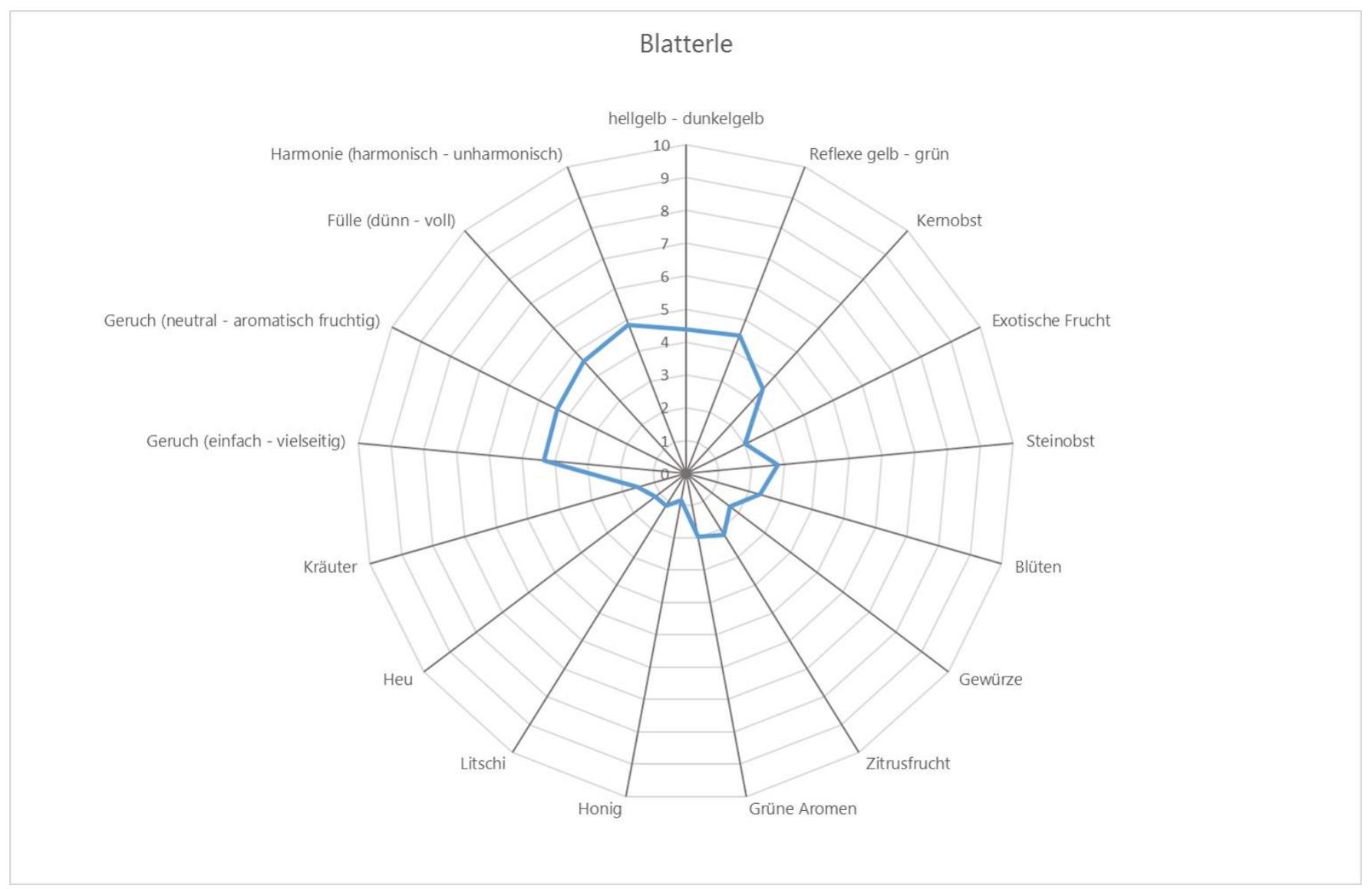

Abb. 2: Netzdiagramm Blatterle // radar chart Blatterle. 


\section{Blatterle}

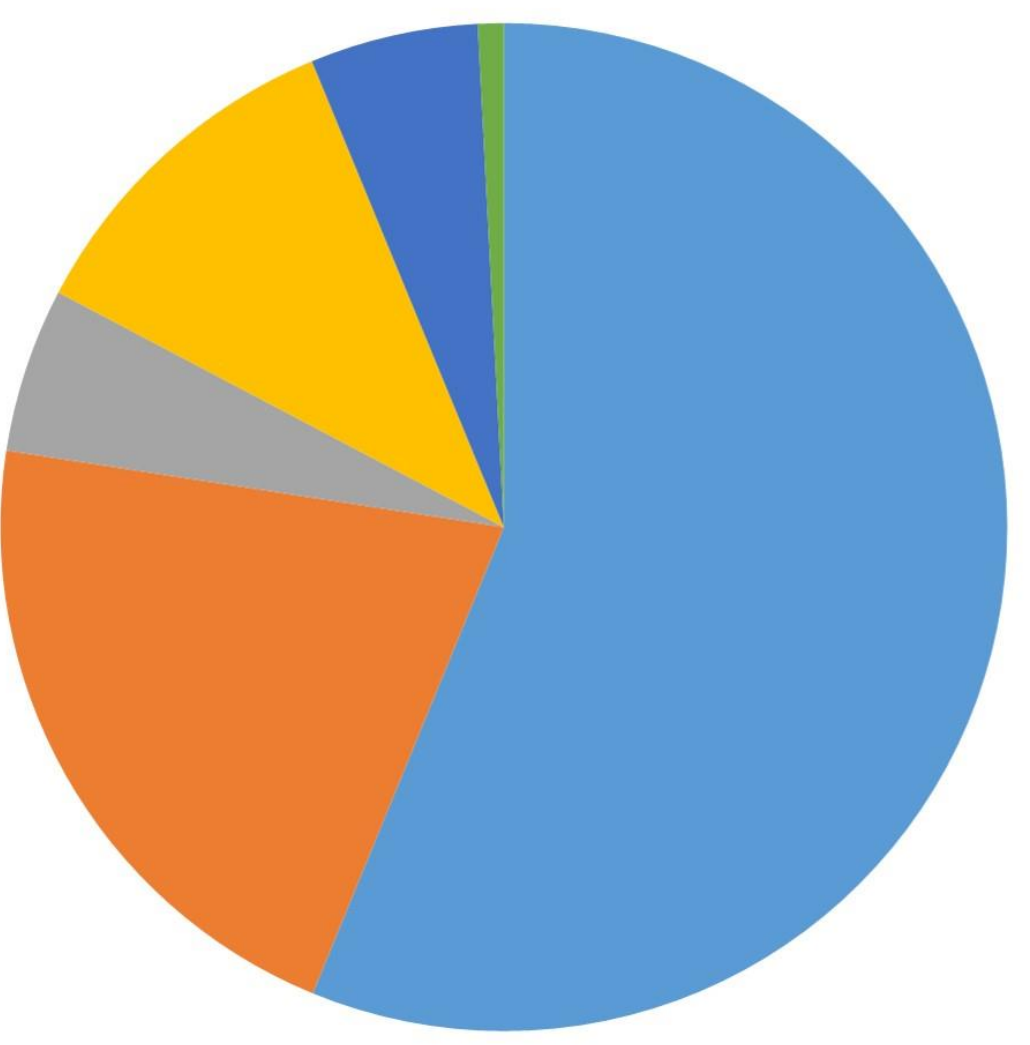

- Burgundertyp

- Rieslingtyp

Gewürztraminertyp

Sauvignontyp

- Muskattyp

- Andere

Abb. 3: Tortendiagramm Blatterle // pie chart Blatterle. 


\section{Fraueler}

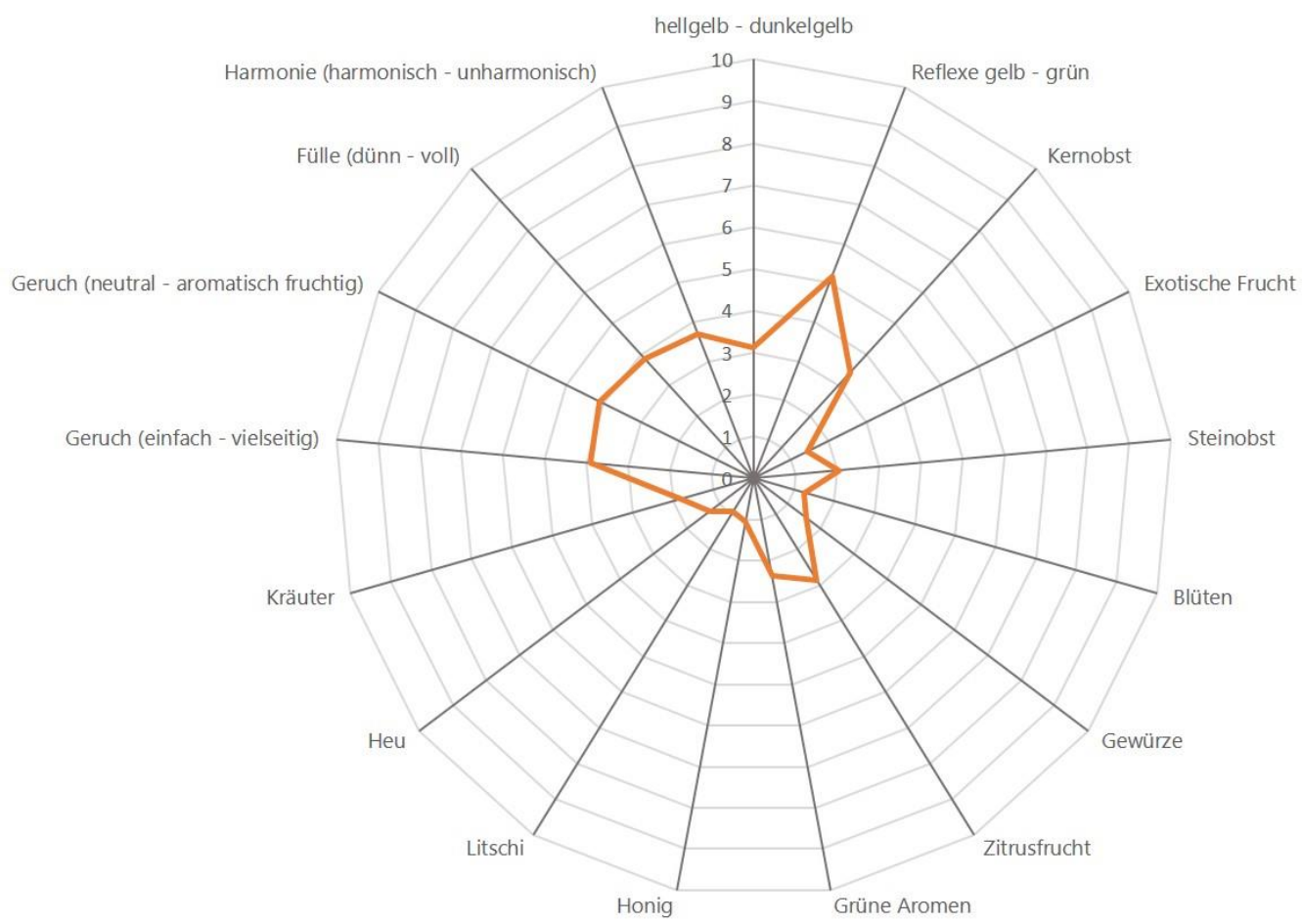

Abb. 4: Netzdiagramm Fraueler // radar chart Fraueler. 


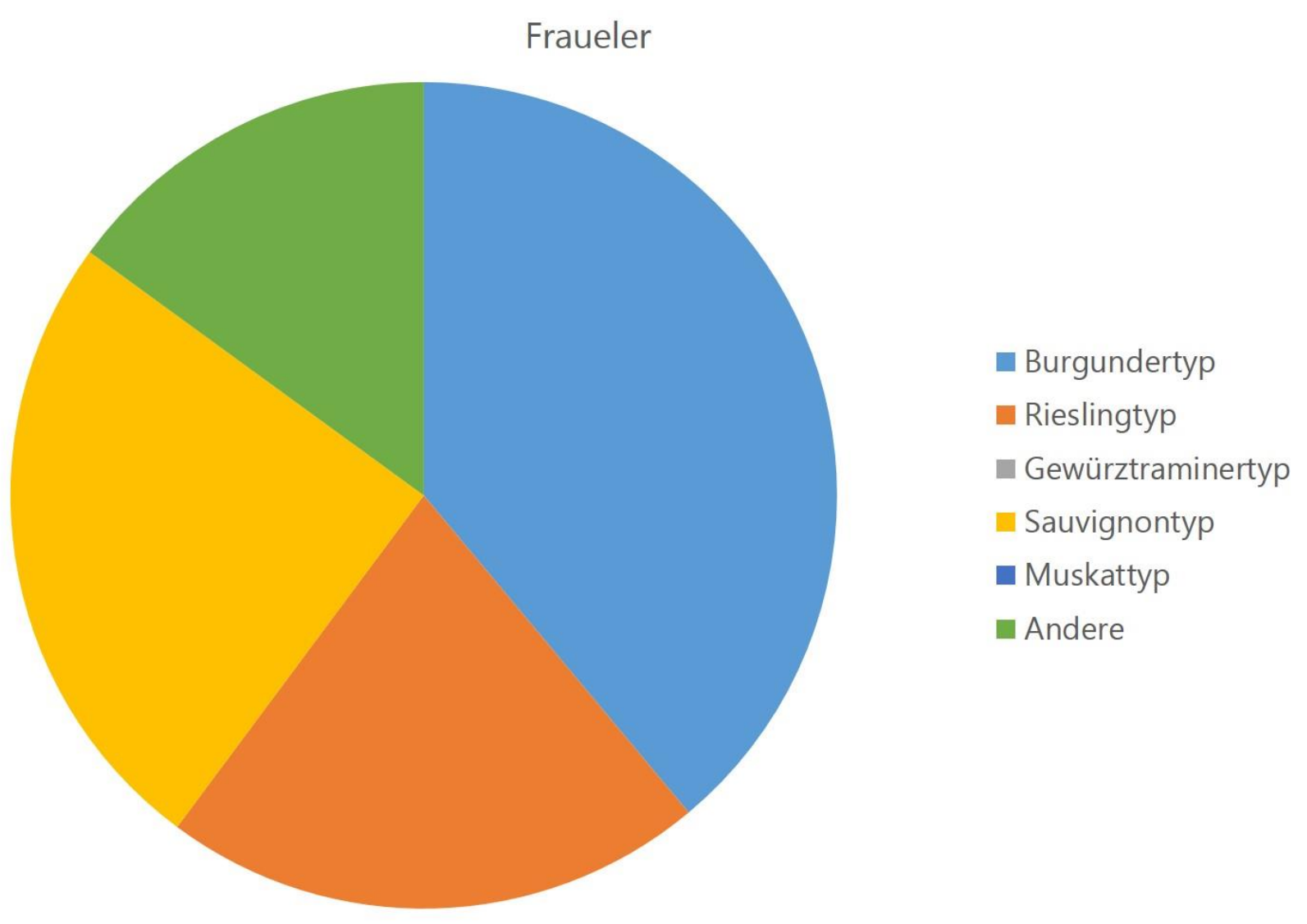

Abb. 5: Tortendiagramm Fraueler // pie chart Fraueler. 


\section{Weissterlaner}

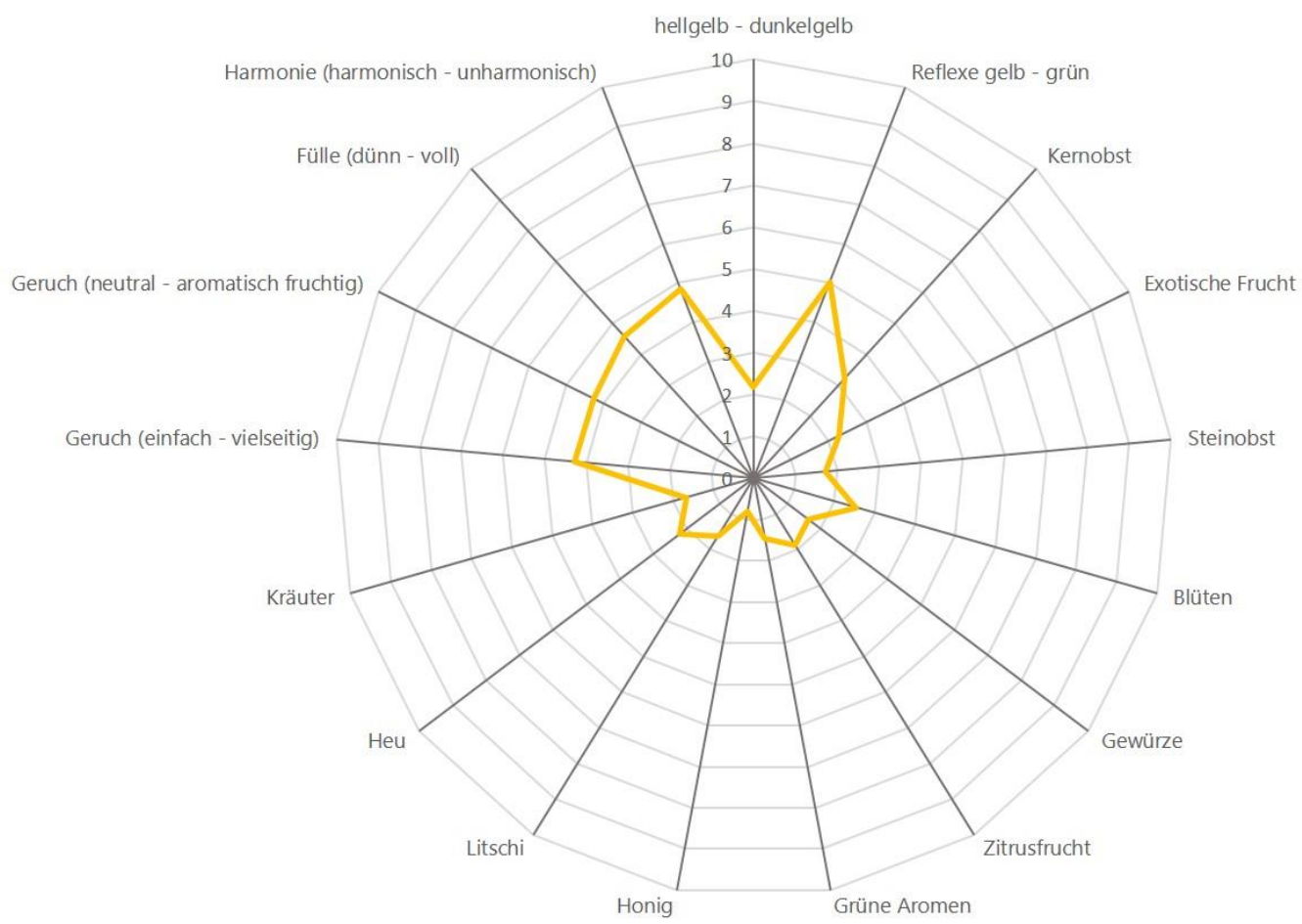

Abb. 6: Netzdiagramm Weissterlaner // radar chart Weissterlaner. 


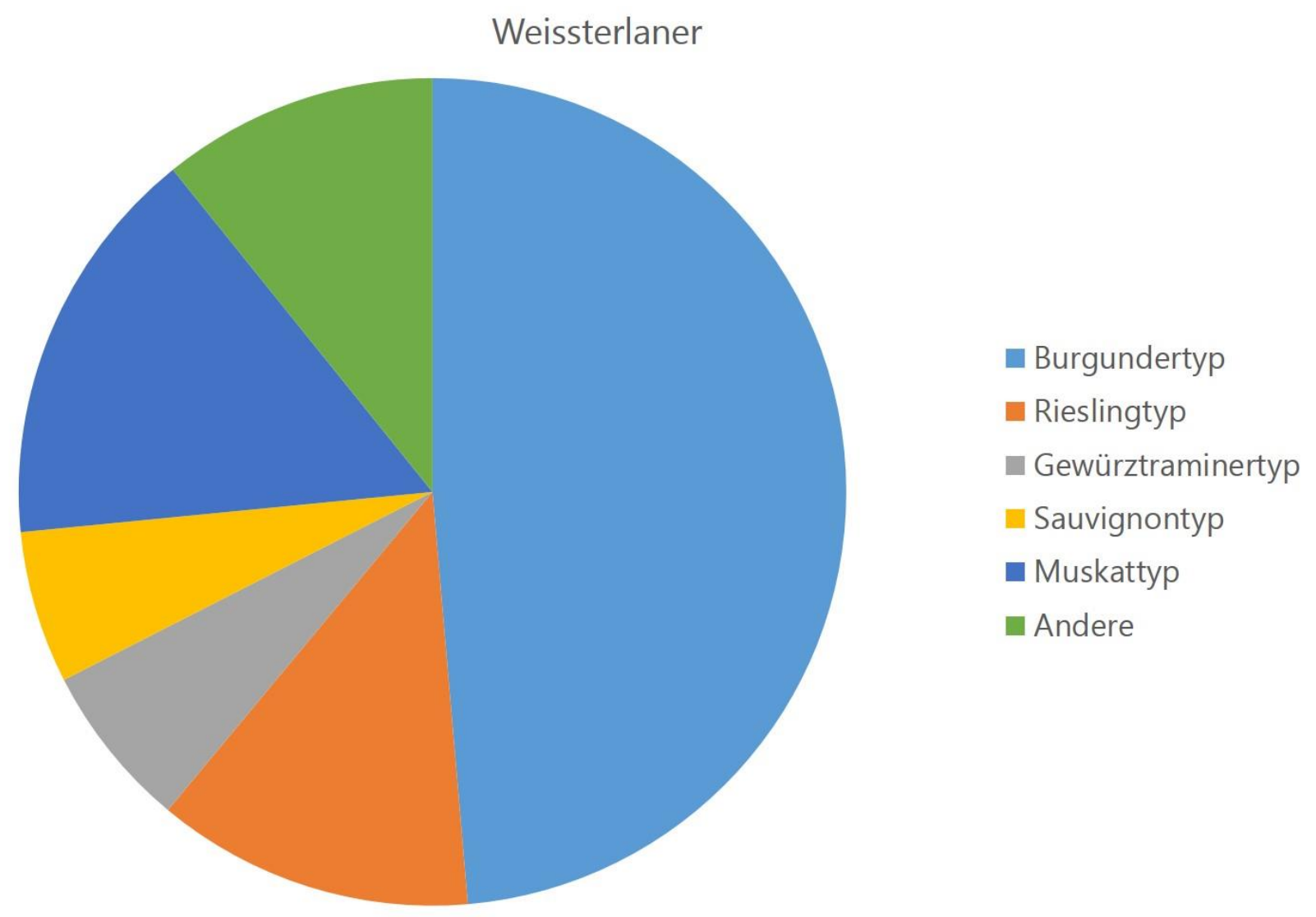

Abb. 7: Tortendiagramm Weissterlaner // pie chart Weissterlaner. 


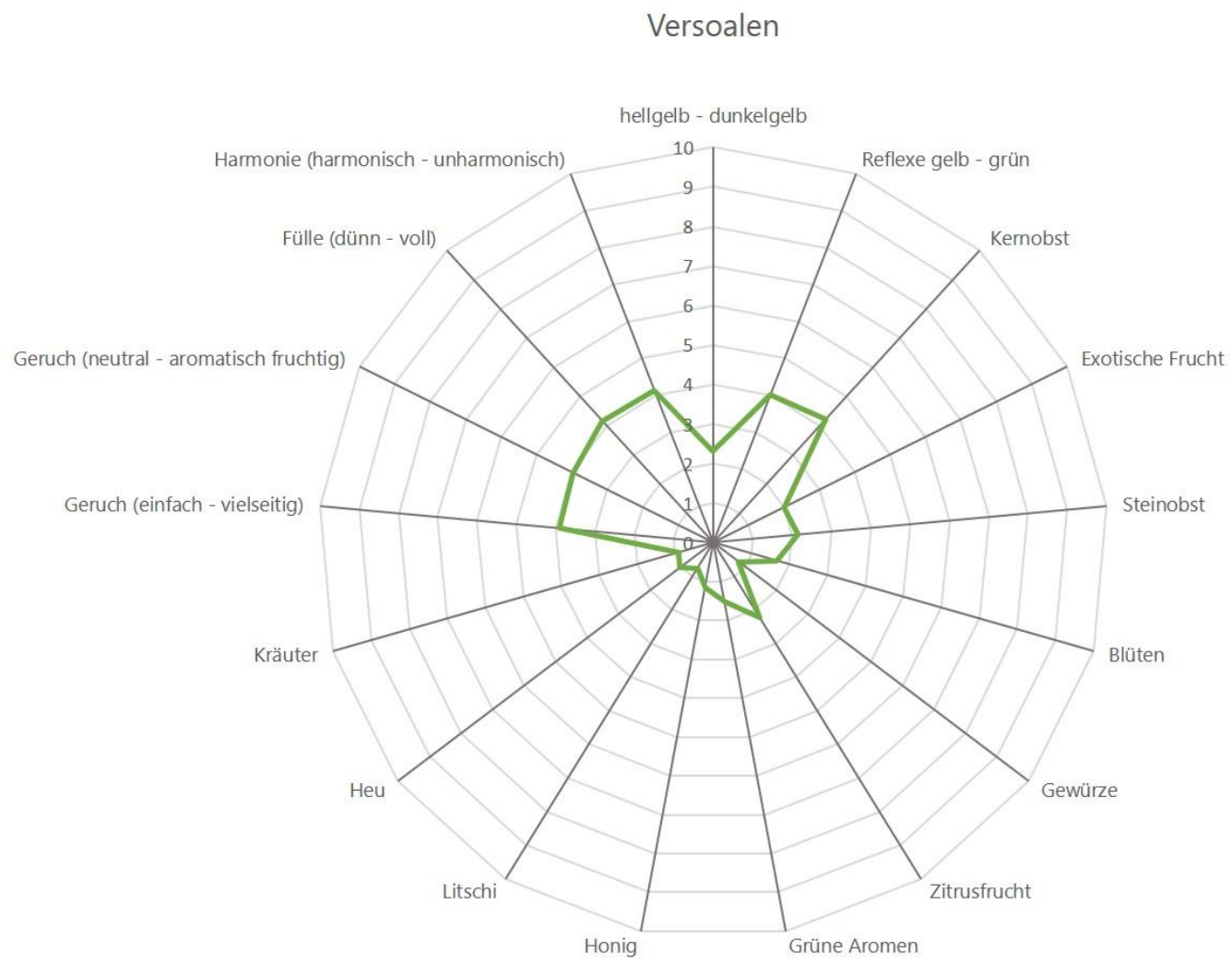

Abb. 8: Netzdiagramm Versoalen // radar chart Versoalen. 


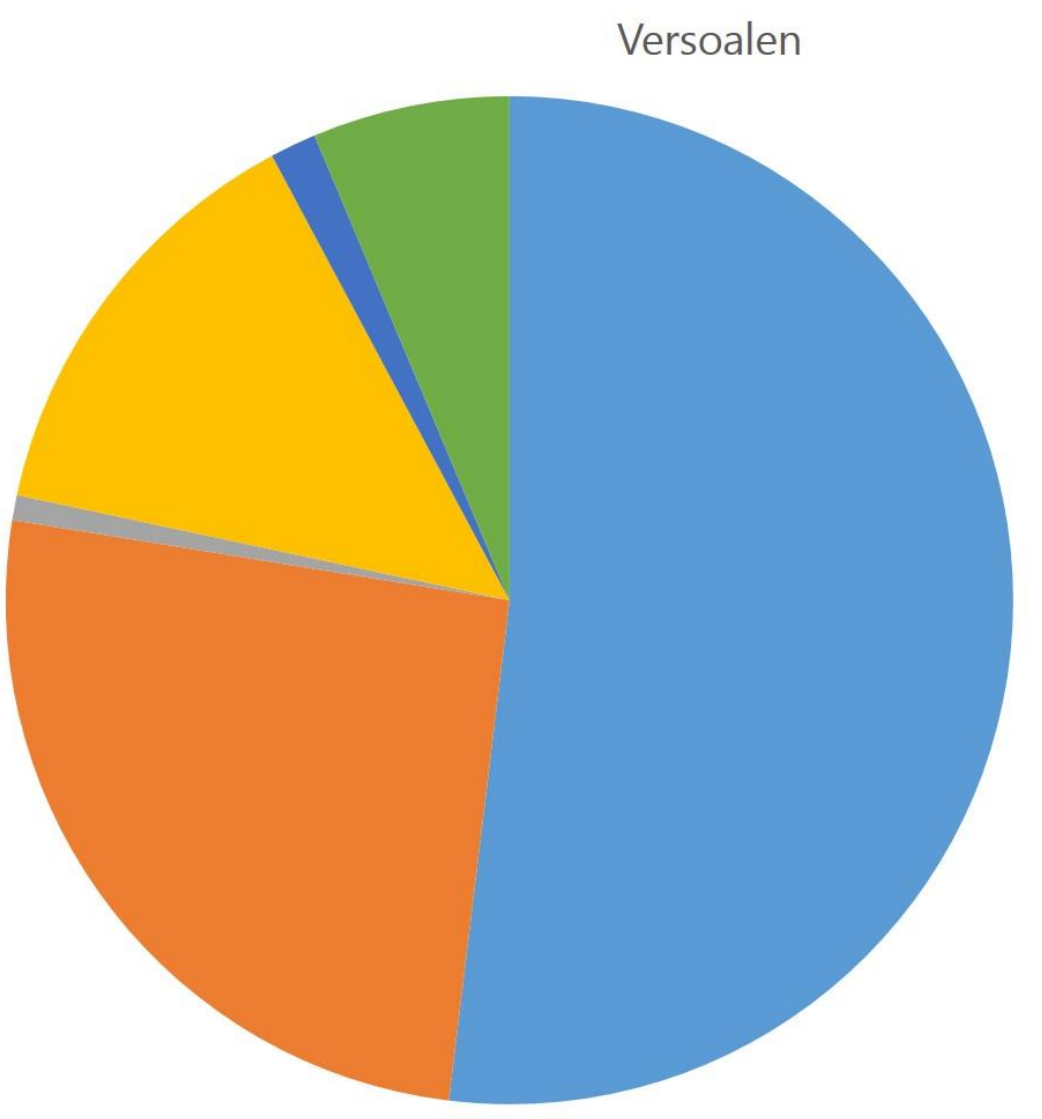

Burgundertyp

- Rieslingtyp

- Gewürztraminertyp

- Sauvignontyp

- Muskattyp

andere

Abb. 9: Tortendiagramm Versoalen // pie chart Versoalen. 


\section{Furner}

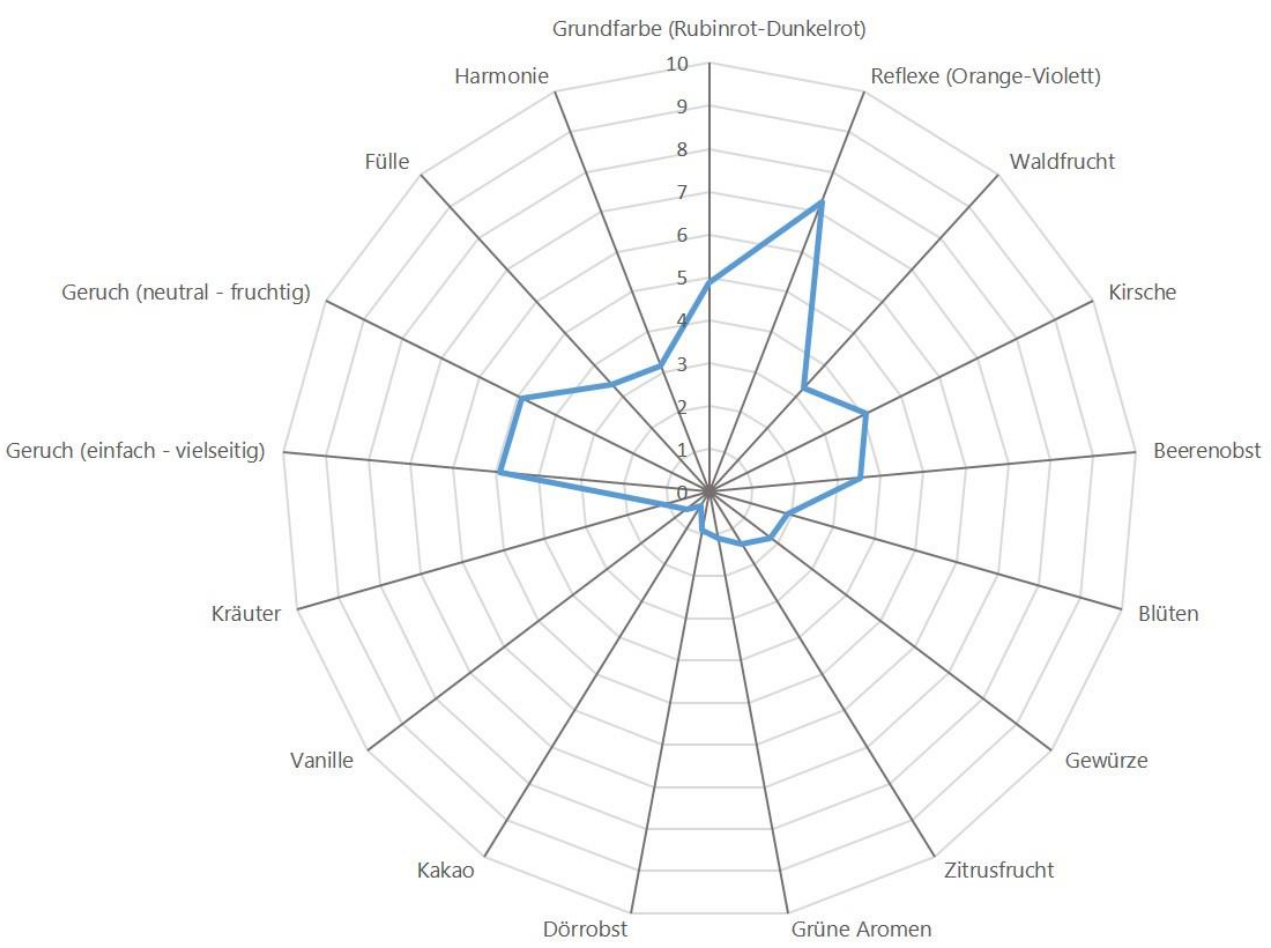

Abb. 10: Netzdiagramm Furner // radar chart Furner. 


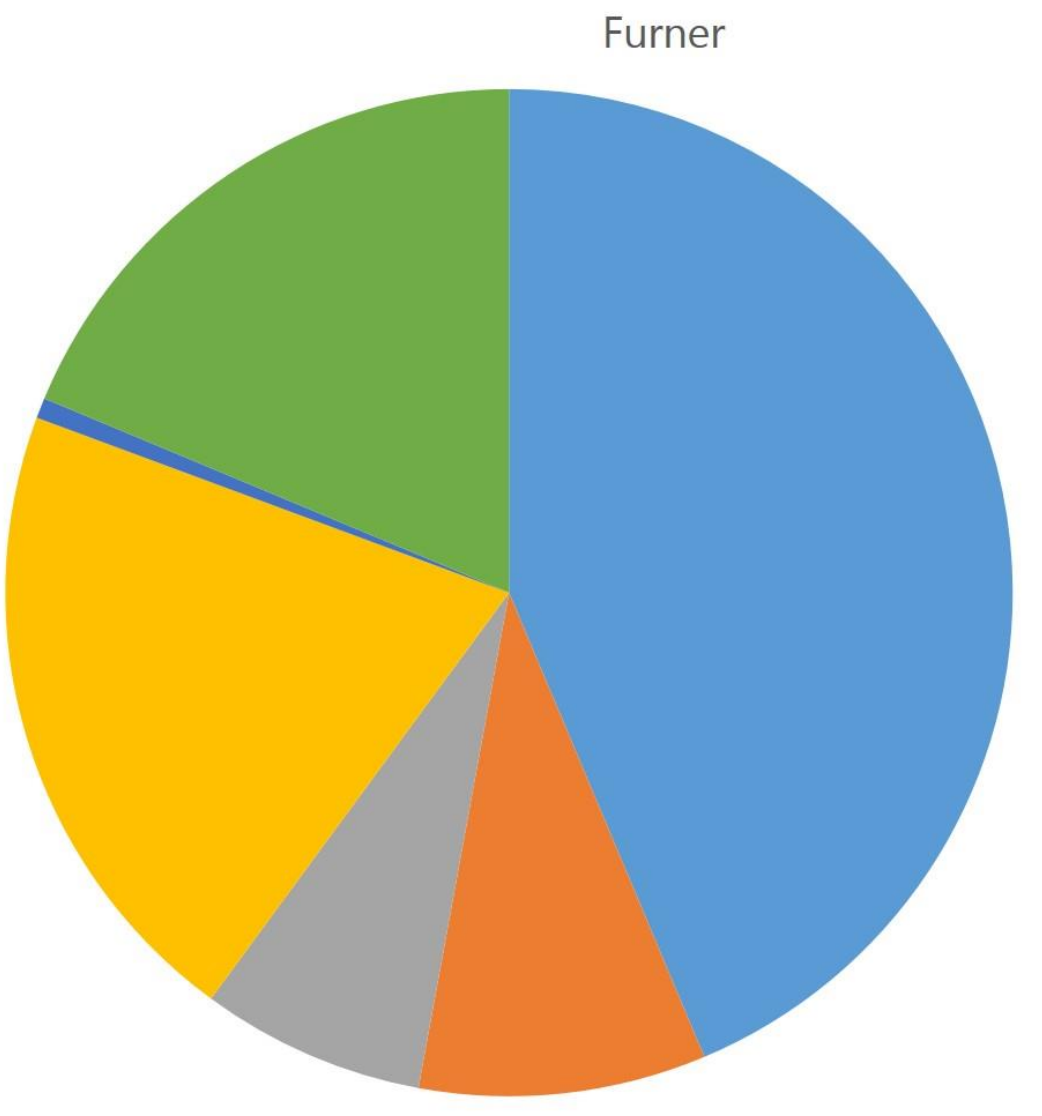

- Vernatschtyp

- Merlottyp

- Lagreintyp

- Burgundertyp

- Cabernettyp

- Andere

Abb. 11: Tortendiagramm Furner // pie chart Furner. 
Vergleich von verschiedenen Rebsorten über die Gerbstoffquantität im Wein

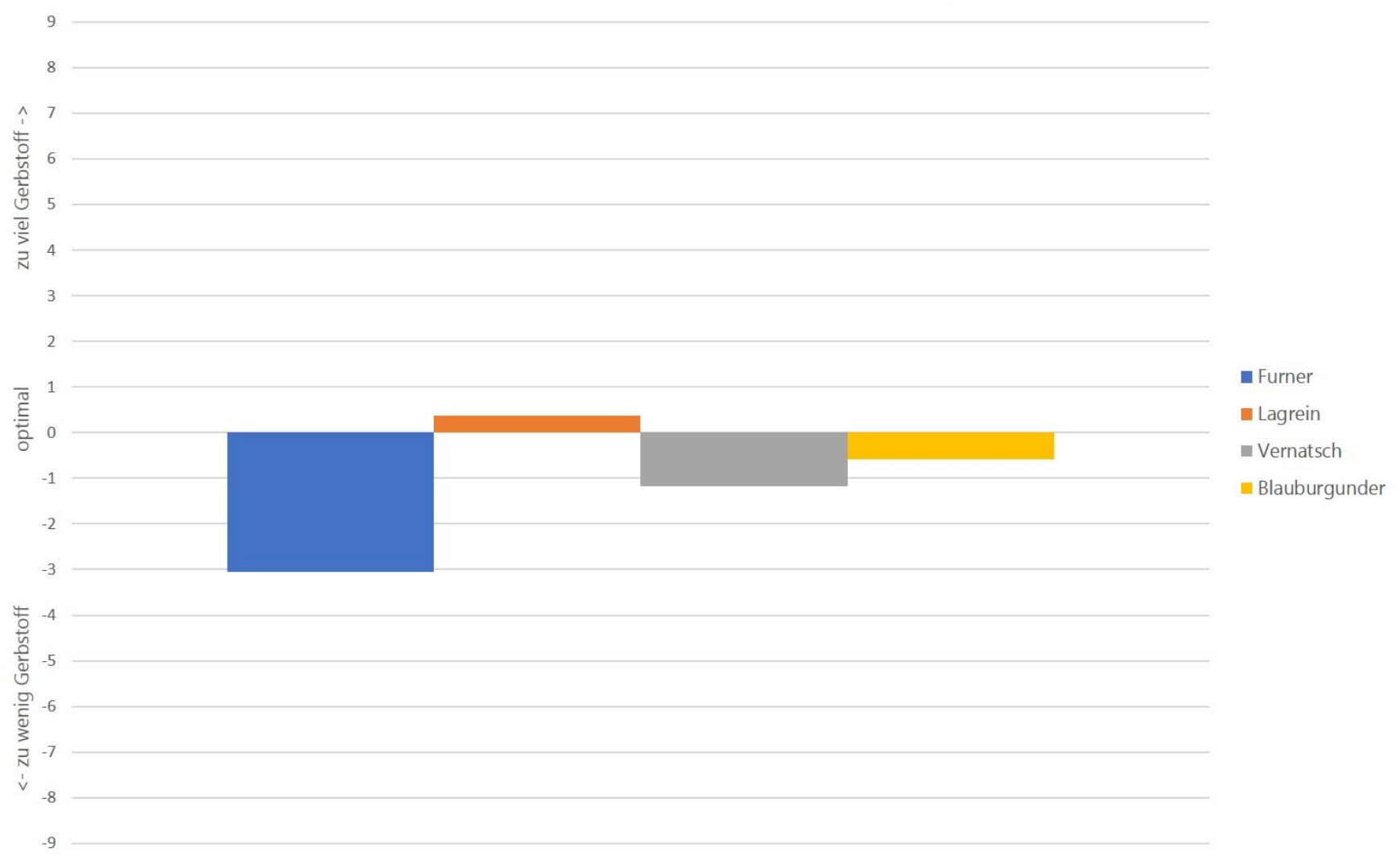

Abb. 12: Gerbstoffquantität von Rotweinsorten // tannin quantity of redwine varieties. 


\section{ANHANG 2: TABELLEN}

Tab. 1: Übersicht über die durchgeführten Anreicherungen des Alkoholgehaltes // Overview of the enrichment of the alcohol content.

$\begin{array}{ccccc}\text { Jahr } & \text { Versoalen } & \text { Weissterlaner } & \text { Blatterle } & \text { Fraueler } \\ 2014 & +1 \% \text { vol. effektiv } & +1 \% \text { vol. effektiv } & - & +1 \% \text { vol. effektiv } \\ 2015 & +1.3 \% \text { vol. effektiv } & +1.3 \% \text { vol. effektiv } & +1.3 \% \text { vol. effektiv } & - \\ 2016 & - & - & - & +1.5 \% \text { vol. effektiv }\end{array}$


Tab. 2: Lesezeitpunkte // Harvest dates.

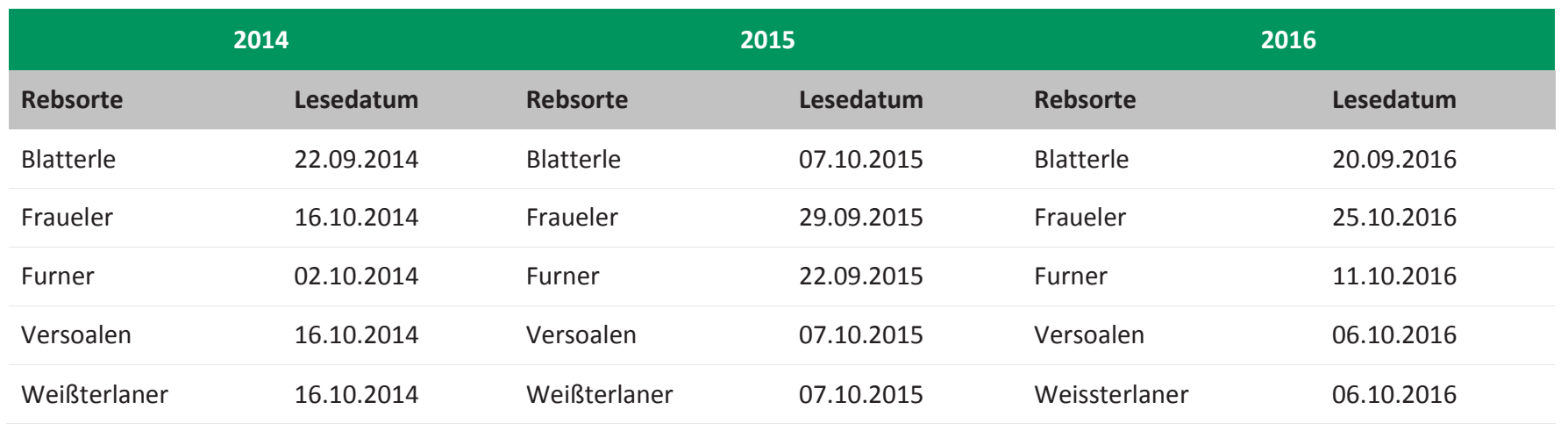


Tab. 3: Diskriminierenden Kriterien der Verkostung von Blatterle, Versoalen, Weissterlaner und Fraueler. // Discriminatory criteria of the tasting of Blatterle, Versoalen, Weissterlaner and Fraueler.

Jahrgang 2014+2015

Beschreibung

Kriterium

hellgelb-dunkelgelb

Grundfarbe

Reflexe

gelb-grün

Kernobst

Exotische Frucht

Steinobst

Blüten

Gewürze

Zitrusfrucht

Grüne Aromen

Honig

Litschi

$\mathrm{Heu}$

Kräuter

Geruch

Geruch+

Fülle

Harmonie nicht vorhanden-vorhanden

nicht vorhanden-vorhanden

nicht vorhanden-vorhanden

nicht vorhanden-vorhanden

nicht vorhanden-vorhanden

nicht vorhanden-vorhanden

nicht vorhanden-vorhanden

nicht vorhanden-vorhanden

nicht vorhanden-vorhanden

nicht vorhanden-vorhanden

nicht vorhanden-vorhanden

einfach-vielseitig

neutral-aromatisch fruchtig

dünn-voll

unharmonisch-harmonisch
Jahrgang 2016

Beschreibung

$--$

$--$

nicht vorhanden-vorhanden

nicht vorhanden-vorhanden

nicht vorhanden-vorhanden

nicht vorhanden-vorhanden

nicht vorhanden-vorhanden

nicht vorhanden-vorhanden

nicht vorhanden-vorhanden

nicht vorhanden-vorhanden

nicht vorhanden-vorhanden

einfach-vielseitig

neutral-aromatisch fruchtig

dünn-voll

unharmonisch-harmonisch 
Tab. 4: Diskriminierenden Kriterien der Verkostung von Furner // Discriminatory criteria of the tasting of Furner.

\begin{tabular}{|c|c|c|}
\hline & Jahrgang 2014+2015 & Jahrgang 2016 \\
\hline Kriterium & Beschreibung & Beschreibung \\
\hline Grundfarbe & rubinrot-dunkelrot & --- \\
\hline Reflexe & orange/braun-violett & --- \\
\hline Waldfrucht & nicht vorhanden-vorhanden & nicht vorhanden-vorhanden \\
\hline Kirsche & nicht vorhanden-vorhanden & nicht vorhanden-vorhanden \\
\hline Beerenobst & nicht vorhanden-vorhanden & nicht vorhanden-vorhanden \\
\hline Blüten & nicht vorhanden-vorhanden & nicht vorhanden-vorhanden \\
\hline Gewürze & nicht vorhanden-vorhanden & nicht vorhanden-vorhanden \\
\hline Zitrusfrucht & nicht vorhanden-vorhanden & nicht vorhanden-vorhanden \\
\hline Grüne Aromen & nicht vorhanden-vorhanden & nicht vorhanden-vorhanden \\
\hline Dörrobst & nicht vorhanden-vorhanden & --- \\
\hline Schokolade & --- & nicht vorhanden-vorhanden \\
\hline Kakao & nicht vorhanden-vorhanden & --- \\
\hline Vanille & nicht vorhanden-vorhanden & --- \\
\hline Kräuter & nicht vorhanden-vorhanden & --- \\
\hline Geruch & einfach-vielseitig & einfach-vielseitig \\
\hline Geruch+ & neutral-aromatisch fruchtig & neutral-aromatisch fruchtig \\
\hline Gerbstoffquantität & zu wenig Gerbstoff-optimal-zu viel Gerbstoff & zu wenig Gerbstoff-optimal-zu viel Gerbstoff \\
\hline Gerbstoff Hart & --- & hart-rund \\
\hline Gerbstoff Bitter & --- & bitter-nicht bitter \\
\hline Gerbstoff Reife & --- & unreif-reif \\
\hline Fülle & dünn-voll & dünn-voll \\
\hline Harmonie & unharmonisch-harmonisch & unharmonisch-harmonisch \\
\hline Gesamteindruck & --- & schlecht-gut \\
\hline
\end{tabular}


Tab. 5: Deskriptive Statistik Blatterle // Descriptive statistics Blatterle.

\begin{tabular}{lccccc} 
& Deskriptive Statistik "Blatterle" & & \\
& N & Minimum & Maximum & Mittelwert & Standardabweichung \\
\hline Alkohol (\%vol.) & 6 & 11.67 & 13.32 & 12.5733 & 0.62433 \\
\hline pH-Wert & 6 & 3.35 & 3.55 & 3.45 & 0.07403 \\
\hline Gesamtsäure (g/l) & 6 & 4.66 & 7.15 & 5.6517 & 1.06359 \\
\hline Gesamtextrakt (g/l) & 6 & 19.53 & 23 & 20.71 & 1.60431 \\
\hline Gesamtpolyphenole nach Folin (mg/l) & 6 & 203 & 258 & 224.5 & 22.793 \\
\hline Weinsäure (g/l) & 6 & 1.71 & 2.5 & 2.1467 & 0.33399 \\
\hline Apfelsäure (g/l) & 6 & 1.38 & 3.12 & 2.2017 & 0.71373 \\
\hline Gültige Werte (Listenweise) & 6 & & & &
\end{tabular}


Tab. 6: Deskriptive Statistik Fraueler // Descriptive statistics Fraueler.

\begin{tabular}{|c|c|c|c|c|c|}
\hline \multicolumn{6}{|c|}{ Deskriptive Statistik "Fraueler" } \\
\hline & $\mathbf{N}$ & Minimum & Maximum & Mittelwert & Standardabweichung \\
\hline Alkohol (\%vol.) & 6 & 11.59 & 13.61 & 12.3417 & 0.84552 \\
\hline $\mathrm{pH}$-Wert & 6 & 2.98 & 3.11 & 3.0317 & 0.05115 \\
\hline Gesamtextrakt (g/l) & 6 & 20.42 & 26.41 & 22.9583 & 2.5394 \\
\hline Gesamtpolyphenole nach Folin (mg/I) & 6 & 183 & 244 & 214 & 24.347 \\
\hline Weinsäure (g/l) & 6 & 2.58 & 3.4 & 2.9183 & 0.29267 \\
\hline Apfelsäure (g/l) & 6 & 2.78 & 4.58 & 3.6017 & 0.68488 \\
\hline Gültige Werte (Listenweise) & 6 & & & & \\
\hline
\end{tabular}


Tab. 7: Deskriptive Statistik Weissterlaner // Descriptive statistics Weissterlaner.

\begin{tabular}{lccccc} 
& Deskriptive Statistik "Weissterlaner" & & \\
& N & Minimum & Maximum & Mittelwert & Standardabweichung \\
\hline Alkohol (\%vol.) & 4 & 11.85 & 12.96 & 12.2625 & 0.51169 \\
\hline pH-Wert & 4 & 3.3 & 3.47 & 3.365 & 0.07326 \\
\hline Gesamtsäure (g/l) & 4 & 5.08 & 6.09 & 5.5175 & 0.42161 \\
\hline Gesamtextrakt (g/l) & 4 & 18.74 & 21.55 & 19.495 & 1.37192 \\
\hline Gesamtpolyphenole nach Folin (mg/l) & 4 & 199 & 288 & 239.25 & 37.241 \\
\hline Weinsäure (g/l) & 4 & 1.62 & 2.58 & 2.1925 & 0.46414 \\
\hline Apfelsäure (g/l) & 4 & 0.75 & 2.11 & 1.6075 & 0.59197 \\
\hline Gültige Werte (Listenweise) & 4 & & & &
\end{tabular}


Tab. 8: Deskriptive Statistik Versoalen // Descriptive statistics Versoalen.

\begin{tabular}{lccccc} 
& Deskriptive Statistik "Versoalen" & & \\
& N & Minimum & Maximum & Mittelwert & Standardabweichung \\
\hline Alkohol (\%vol.) & 6 & 11.42 & 13.63 & 12.1767 & 1.08437 \\
\hline pH-Wert & 6 & 3.04 & 3.35 & 3.1867 & 0.11553 \\
\hline Gesamtsäure (g/l) & 6 & 5.48 & 8.76 & 7.2 & 1.36245 \\
\hline Gesamtextrakt (g/l) & 6 & 18.03 & 22.8 & 20.8767 & 2.10681 \\
\hline Gesamtpolyphenole nach Folin (mg/l) & 6 & 186 & 247 & 204.67 & 22.115 \\
\hline Weinsäure (g/l) & 6 & 2.36 & 2.72 & 2.5583 & 0.14538 \\
\hline Apfelsäure (g/l) & 6 & 1.75 & 3.93 & 2.825 & 0.89054 \\
\hline Gültige Werte (Listenweise) & 6 & & & &
\end{tabular}


Tab. 9: Deskriptive Statistik Furner // Descriptive statistics Furner.

\begin{tabular}{|c|c|c|c|c|c|}
\hline \multicolumn{6}{|c|}{ Deskriptive Statistik "Furner" } \\
\hline & $\mathbf{N}$ & Minimum & Maximum & Mittelwert & Standardabweichung \\
\hline Alkohol (\%vol.) & 6 & 10.72 & 11.76 & 11.1183 & 0.42348 \\
\hline pH-Wert & 6 & 3.25 & 3.37 & 3.3167 & 0.03983 \\
\hline Gesamtextrakt (g/l) & 6 & 23.77 & 24.9 & 24.3133 & 0.37087 \\
\hline Gesamtpolyphenole nach Folin (mg/l) & 6 & 472 & 1312 & 774.33 & 344.886 \\
\hline Anthocyane (mg/l) & 6 & 130 & 205 & 166 & 29.819 \\
\hline Weinsäure (g/I) & 6 & 2.23 & 2.68 & 2.4117 & 0.18798 \\
\hline Milchsäure (g/l) & 6 & 2.89 & 3.51 & 3.1983 & 0.23962 \\
\hline Gültige Werte (Listenweise) & 6 & & & & \\
\hline
\end{tabular}

\section{(a) (1) ब (8)}

Dieses Werk ist lizenziert unter einer Creative Commons Namensnennung - Nicht kommerziell 4.0 International Lizenz.

Quest'opera è distribuita con Licenza Creative Commons Attribuzione - Non commerciale 4.0 Internazionale.

This work is licensed under a Creative Commons Attribution - NonCommercial 4.0 International License.

Für alle Abbildungen und Tabellen ohne Nennung des Urhebers gilt: (C) Versuchszentrum Laimburg.

Per tutte le immagini e tabelle senza menzione dell'artefice vale: (C) Centro di Sperimentazione Laimburg.

For all figures and tables without mention of the originator applies: (c) Laimburg Research Centre. 